\title{
The hard problem of design
}

\author{
SEAN HANNA \\ Bartlett School of Graduate Studies, University College London, London, United Kingdom
}

It has often been said of artificial intelligence (AI) that once an essential aspect of intelligence has been emulated, it ceases to remain AI. Logical reasoning, chess playing, and to some extent even natural language processing have over the years become simple stock algorithms, which are seemingly well understood. Our human ability for design seems resilient to this fate, and yet the difficulty of the tasks we face in engineering indicates the clear need for intelligent computation as an essential aid. The importance of a journal like $A I$ EDAM should be evident in the range of factors that make design, analysis, and manufacturing increasingly complex. The more globally connected world of the present means that the task of design is more distributed, often between teams in different continents. Manufacturing similarly occurs in geographically distant locations, with first data and then physical components transported internationally. The demand for better performance and better degrees of optimization to save limited resources of energy or materials demands nonstandard solutions. The size and complexity of our products, including whole cities or urban regions designed in their entirety and constructed almost overnight, is unprecedented. All of these factors stretch the bounds of normal human intuition and traditional methods, so a need for AI in engineering is today perhaps greater than ever before.

When AI EDAM began, Rittel and Weber (1984) had recently described design problems as "wicked"-unique, unrepresentable, and boundless in scale-but the past 25 years have seen changes to our ideas of computation that may yet help us to tackle them. This speculation is admittedly optimistic, but by way of suggesting some direction for the future of our field, here are three observations from its past.

As the first volume of AI EDAM was going to press, Rodney Brooks suggested (although this was not published until 1991) that conventional models of complex events were inherently limited, and it is "better to use the world as its own model." Clear representation, in many circles, would no longer be a primary focus of AI.

Google is presently celebrating its 13 th birthday, putting its origin at about the midpoint of AI EDAM's history. Although

Reprint requests to: Sean Hanna, Bartlett School of Graduate Studies, University College London, Gower Street, London WC1E 6BT, UK. E-mail: s.hanna@ucl.ac.uk it is obvious that our ability to search the Internet has changed the way we work, what is perhaps of even broader impact is the necessary development of new algorithms and techniques to deal with massive amounts of relatively unstructured data. Internet search developers are just not interested in anything less than data in the billions or trillions. This necessitates a change from simple representations and a change in how we make sense of information, as some meaning is extracted from all of the mess that exists out there.

In the present, thanks to Moore's law (1965), computers are 5000 times faster than 25 years ago, but even more crucial are structural changes in computing architecture. Graphics rendering in hardware, which are due mainly to the video games industry, exceeds what Moore's law alone could provide for software rendering. The result is real time rendering that allows for immediate feedback when dealing with understanding of surface geometry, light, and so forth. This completely changes the nature of how the computer can be used in design by making results accessible to our intuition. What about fluid dynamics? What about pedestrian simulation and more complex human behavior via agent-based models? It is also relevant to the games industry that these should also become more immediate and familiar in the next few years, changing the nature of design yet again.

These events are significant because engineering has too often been considered to trade in clear, standardized representations, to deal with well-structured problems, and to favor method over intuition. Yet where the design task is difficult and real intelligence and creativity are needed, this is not the case. The above changes to representation, algorithm, and hardware are perhaps only now becoming ubiquitous enough to make their full impact on engineering practice. However, if this results in a better understanding of how we can deal with our more difficult problems, the coming years of $A I E D A M$ should be a very exciting period.

\section{REFERENCES}

Brooks, R.A. (1991). Intelligence without representation. Artificial Intelligence 47, 139-159.

Moore, G.E. (1965). Cramming more components onto integrated circuits Electronics 38(8).

Rittel, H.W.J., \& Webber, M.M. (1984). Planning problems are wicked problems. In Developments in Design Methodology (Cross, N., Ed.). New York: Wiley. 